\title{
Epidemiology, species distribution and outcome of nosocomial Candida spp. bloodstream infection in Shanghai - A retrospective of 11-year clinical analysis in a shanghai tertiary hospital
}

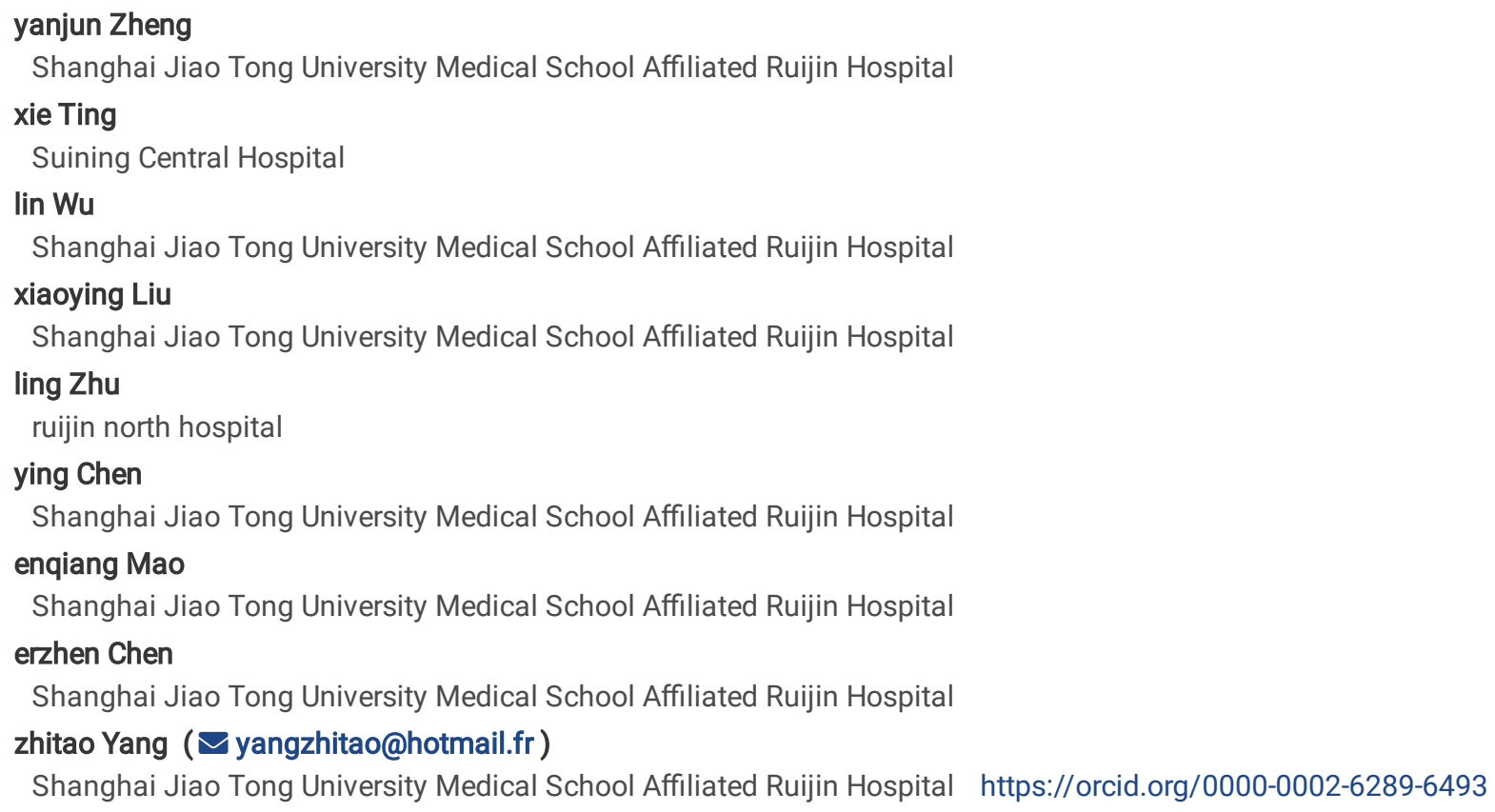

\section{Research article}

Keywords: Candida spp; Bloodstream infection; Epidemiology; Species distribution; Antifungal therapy; Susceptibility; Early treatment; Survival

Posted Date: February 27th, 2020

DOI: https://doi.org/10.21203/rs.2.24729/v1

License: (a) This work is licensed under a Creative Commons Attribution 4.0 International License. Read Full License 


\section{Abstract}

Background: Candida spp. are important opportunist pathogens causing bloodstream infections (BSIs). The present study aims to describe the current epidemiology of Candida BSI in a large Shanghai Tertiary-care Hospital from 2008 to 2018, and to identify the risk factors and the impact of antifungal therapy on clinical outcome.

Methods: From January 2008 to December 2018, all consecutive patients who developed Candida BSI at Ruijin Hospital were enrolled. Underlying diseases, clinical severity, species distribution, antifungal therapy and its impact on the outcome were analyzed.

Results: The incidence of nosocomial Candida BSI was 0.39 episodes/1000 hospitalized patients, and overall 30-day mortality rate was $28.5 \%$. Among the 393 cases of Candida BSI, 299 cases (76.1\%) received antifungal therapy. 247 received appropriate antifungal therapy, and 52 received delayed antifungal therapy (5 days after onset of Candida BSI). The 30-day mortality rate was significantly lower in those who received appropriate antifungal therapy or delayed antifungal therapy compared with those who did not receive antifungal therapy (25.5\% and $23.1 \%$ vs. $39.4 \% \bigotimes \mathrm{P}=0.012$ and $\mathrm{P}=0.046$ ). In multivariate Cox regression analysis, age, chronic renal failure, mechanical ventilation, neutropenia were independent risk factors for the 30-day mortality rate, while antifungal therapy was a protective factor for short-term survival rate.

Conclusion: The epidemiology of Candida BSI in Shanghai differed from that observed in Western countries. Antifungal therapy did influence the short-term survival, while there was no significant difference between the mortality for those who received appropriate antifungal therapy and for those who received delayed antifungal therapy.

\section{Background}

The incidence of invasive fungal infection has increased over time, especially for candida bloodstream infections (BSIs), which was associated with considerable excess mortality and costs. In the past two decades, the incidence of fungal infection has increased from 0.1 episodes/1000 admissions to 0.3-0.6 episodes/1000 admissions in China, North America and some European countries[1-4]. The mortality rate was as high as $47.9 \%[5]$. The lack of specific clinical manifestations, rapid and sensitive diagnostic tests has led to the fact that patients were hard to get access to early diagnosis and treatment. The overuse of antifungal agent results in increased costs, selection pressure for resistance and adverse drug interactions, etc. Several studies showed that delayed antifungal therapy (more than $48 \mathrm{~h}$ from onset) was associated with higher mortality[6], while others have yielded conflicting results[7-9].

In this study, we retrospectively analyzed all Candida bloodstream infection patients from 2008 to 2018 in our hospital, aiming to describe their clinical characteristics, species distribution, antifungal therapy and to identify its risk factors for 30-day mortality.

\section{Methods}

\section{Study Setting and population}

A retrospective analysis of consecutive Candida bloodstream episodes in adults collected from the microbiology database of a 1900-bed teaching hospital in Shanghai, was performed over a 11-year period (from $1^{\text {st }}$ January 2008 to $31^{\text {st }}$ December 2018).

Demographics, underlying diseases, co-morbidities, severity of clinical features, Candida species distribution, prior antibiotic use and appropriate or delayed antifungal treatment were compared among the patients with Candida BSI. The initial and target antifungal agent use was also collected.

A case of Candida BSI was defined as a patient with at least one blood culture positive for a Candida. Two or more blood cultures positive to different Candida within 14 days were considered one episode of Candida BSI[10]. Neutropenia was defined as $<500 / \mathrm{mm}^{3}$ absolute neutrophil count. Prior corticosteroid was defined as receiving $>1 \mathrm{mg} / \mathrm{kg} / \mathrm{d}$ prednisone for more than one week or equivalent before Candida BSI onset. Antibiotic treatment was considered if the patient has been on antibiotic treatment prior to Candida BSI, for at least 3 days.

\section{Laboratory Methods}

Isolates were detected from blood cultures using the BACTEC ${ }^{T M}$ FX system (Becton Dickinson, Inc., Sparks, MD, USA). Flucytosine, amphotericin B, fluconazole, voriconazole, and itraconazole susceptibility testings were performed using the ATB® FUNGUS 3 system (BioMérieux, France), which was wildly used in China[11], providing susceptibility to antifungals results markedly concordant with those obtained using CLSI and EUCAST methodologies[12].

Initial appropriate antifungal treatment was considered when the appropriate drug (based on subsequent in vitro susceptibility results) with adequate dosage was started within 5 days the first blood culture performed[13]. Adequate dosage of antifungal agent was defined according 
to IDSA 2009 guidelines or 2016 IDSA[10, 14]. Delayed antifungal treatment was defined when appropriate target treatment started after 5 days of BSI onset based on susceptibility results, regardless of whether inappropriate antifungal treatment has been initialed or not. Crude mortality was registered after 30 days from the occurrence of Candida BSI.

\section{Statistical analysis}

Descriptive analyses were used for baseline characteristics and subgroup analyses. Continuous variables were expressed as means \pm SD. The Chi-square-test or 2-tailed Fisher Exact-test was used to compare categorical variables. To define risk factors for mortality, multivariate Cox regression analysis and adjusted odds ratio (OR) with $95 \%$ confidence interval (CI) were calculated. Variables that were associated with 30 -day mortality in Cox univariate analyses with a $\mathrm{P}<0.05$ were entered into multivariate analysis. 2-tailed tests of significance at the level of a $\mathrm{P}$ value of $<0.05$ level was used to determine statistical significance. Statistical analysis has been conducted by using the program SPSS 22.0 .

\section{Ethics}

The study was approved by the local institutional review board (Ruijin Hospital, Shanghai Jiaotong University, School of medicine) and written patient consent was not required because of the observational nature of this study.

\section{Results}

\section{Incidence and demographic characteristics of patients}

A total of 393 consecutive episodes of Candida BSI were collected, occurred in 370 inpatients, during an 11-year study period with an incidence of 0.39 episodes/1000 admissions, which has increased steadily, as shown in Figure 1. (0.21 episodes per 1,000 admissions in $2008,0.24$ in $2009,0.26$ in 2010, 0.44 in 2011, 0.42 in 2012, 0.37 in 2013, 0.40 in 2014, 0.45 in 2015, 0.47 in $2016,0.59$ in 2017 and 0.33 in 2018 ).

The demographic characteristics of the patients are summarized in Table 1. The mean age of patients was $57.6 \pm 19.0$ years and $74.3 \%$ were male. Among 393 episodes, 148 episodes (37.7\%) were from surgical ward, 167 episodes (42.5\%) were from ICU and 78 episodes (19.8\%) were from internal medicine ward, as shown in Figure 2.

C. albicans was isolated in $19.3 \%$ of cases in internal medical wards while in $41.2 \%$ and $38.9 \%$ of cases in surgery wards and ICU, respectively. In contrast, a higher proportion of $C$. tropicalis (34.7\%) was found in internal medicine wards compared to that in surgery wards (21.6\%) and ICUs (21.6\%).

\section{Underlying diseases and clinical features}

The majority of patients with Candida BSI had at least one co-morbidity. 118 (30\%) patients had solid tumors, 48 (12.2\%) patients had hematological malignancies, 77 (19.6\%) patients had diabetes mellitus, 124 (31.6\%) patients had chronic cardiac disease, 52 (13.2\%) patients had chronic pulmonary disease, 42 (10.7\%) patients had chronic renal failure, 26 (6.6\%) patients' skin barrier has been considered compromised, $244(62.1 \%)$ patients had prior surgical intervention, 54 (13.7\%) patients with corticosteroid use, 88 (22.4\%) patients had prior antifungal agents use, 255 (64.9\%) patients received antibiotic prior Candida BSI onset. 244 (72\%) patients had at least two co-morbidities. No patient had human immunodeficiency virus (HIV) infection. Regarding the severity, 309 (78.6\%) patients had fever, 180 (45.8\%) patients received parenteral nutrition, $147(37.4 \%)$ patients received mechanical ventilation, $49(12.5 \%)$ patients received renal replacement therapy, and $42(10.7 \%)$ patients had neutropenia. The clinical characteristics of patients, by Candida species are shown in Table 2.

\section{Candida species and antifungal susceptibility testing}

393 Candida species were isolated in total. 141 (35.9\%) of Candida BSI were C. albicans and 252 (64.1\%) were non-albicans Candida. In nonalbicans Candida sepsis cases, 87 (22.1\%) were caused by C. parapsilosis, 69 (17.6\%) by C. tropicalis, 48 (12.2\%) by C. glabrata, 20 (5.1\%) by C. guilliermondii, $8(2.0 \%)$ by C. sake, 5 (1.3\%) by C.krusei, and 15 by other species (4 C. gum, 3 C. lusitaniae, 2 C. intermedia, 2 C. theae, 2 C.lipolytica, 1 C. famataand 1 C. haemulonii).

Among 393 Candida species, there were 378 episodes with antifungal susceptibility testing results. According to CLSI breakpoints 2012 (CBPs), the rates of susceptibility of C. albicans, C. parapsilosis, C. tropicalis and C. parapsilosis to fluconazole and voriconazole were $94 \%$, $89.5 \%, 52.2 \%, 0 \%$ and $93.3 \%, 91.9 \%, 61.2 \%, 95.8 \%$, respectively. Their susceptibilities to itraconazole were worse (82.1\%, 87. $2 \%, 37.3 \%, 0 \%)$. Amphotericin B and 5-flucytosine remained superior to 95\% susceptibility against common Candida spp., except for C. krusei and C. guilliermondii, which were only $20 \%$ and $47.4 \%$ susceptible to flucytosine, respectively (Table 4 ).

\section{Antifungal therapy and outcome}


Antifungal therapy was administered in 299 (76.1\%) of the cases. 94 (23.9\%) patients did not receive any antifungal treatment. Among the patients who received antifungal therapy, 247 (62.8\%) received appropriate antifungal therapy, and 52 (13.2\%) received delayed antifungal therapy ( 5 days after onset of Candida BSI). Fluconazole was most frequently used as empiric therapy, followed by echinocandins and voriconazole. $18(4.6 \%)$ patients with Candida BSI received a combination therapy.

The overall, 30 -day mortality rate was $28.5 \%$. Mortality rate was significantly higher in internal medicine wards and in ICUs than in surgical wards $(37.2 \%$ and $34.7 \%$ vs. $16.9 \%$, respectively, $\mathrm{P}<0.001)$ (Figure $3 \mathrm{a})$. The mortality for those who received appropriate or delayed antifungal therapy was $25.5 \%$ or $23.1 \%$ ( $P=0.012$ or $P=0.046)$, as compared to $39.4 \%$ for those who hadn't receive any antifungal therapy. However, there was no significant difference between the mortalities for those who received appropriate antifungal therapy and for those who received delayed antifungal therapy (Figure 3a and 3b).

In univariate analysis, age, solid tumor, diabetes mellitus, chronic cardiac disease, chronic renal failure, skin disease, prior surgical intervention, mechanical ventilation, neutropenia, and antifungal therapy were associated with 30-day mortality. In multivariate Cox regression analysis, advanced age $(\mathrm{HR}=1.025 ; 95 \% \mathrm{Cl}, 1.013-1.037 ; \mathrm{P}<0.001)$, chronic renal failure $(\mathrm{HR}=2.018 ; 95 \% \mathrm{Cl} 1.234-3.299 ; \mathrm{P}=0.005)$, mechanical ventilation $(\mathrm{HR}=1.950 ; 95 \% \mathrm{Cl} 1.307-2.912 ; \mathrm{P}=0.001)$, neutropenia $(\mathrm{HR}=4.347 ; 95 \% \mathrm{Cl} 2.462-7.675 ; \mathrm{P}<0.001)$, were independent risk factors for 30-day mortality, while antifungal therapy $(\mathrm{HR}=0.570 ; 95 \% \mathrm{Cl} 0.382-0.849 ; \mathrm{P}=0.006)$ was independent protective factor for 30 -day mortality (Table 3 ).

\section{Discussion}

Our study showed that in our Tertiary-care hospital in Shanghai, the incidence of Candida BSI has increased steadily in the past 11 years. Several studies have shown a substantial increase in the past 2 decades, which was similar to our study[1, 5, 15]. Intensive use of broadspectrum antibiotics may be the main cause. Also, gradually worsening hospitalized patient profiles, underlying co-morbidities including malignancy, and high frequency of surgeries may be predisposing risk factors for increasing incidence.

C. albicans was still the foremost pathogen of Candida BSI. However, in recent years, the trend of increasing rates of non-C. albicans has been reported worldwide, which was also observed in our study (35.9\% for C. albicans. and 64.1\% for non-C. albicans). C. parapsilosis (34.5\%), C. tropicalis (27.4\%) and C. glabrata (19.0\%) account for most of non-C. albicans[16, 17]. In this study, the incidence of C. guilliermondii was incredibly higher than in other studies (4.8\% vs. $0.4 \%)$ [18]. This could be related to the higher rate of parenteral nutrition and surgery (45.8\% and $62.1 \%$ ) in the current study, as some research implies that intravenous nutrition and surgery were significant risk factors for Candida BSI due to C. guilliermondii.[19].

The antifungal susceptibility testing showed that the susceptibility of C. albicans, C. parapsilosis and C. sake for fluconazole was quite high $(94 \%, 89.5 \%, 100 \%)$, while resistance rate of C. tropicalis for fluconazole was as high as $43.3 \%$, which was significantly higher than in other studies abroad[20, 21]. This may deserve more attention from clinicians in actual application.

There were $23.9 \%$ patients with candida BSI didn't receive any antifungal therapy in the present study. It was partly because some patients died before the results of fungal blood cultures can be obtained. Fluconazole was still the most frequently used empirical antifungal therapy, followed by echinocandins. The 30 -day crude mortality rate was $28.5 \%$; a statistically significant finding is that 30 -day mortality rate for Candida BSI in patients from surgical wards was quite low compared with other wards. The contributing factor needs further study.

Another finding in our study was that there was no significant difference in 30-day mortality rate between the patients who received appropriate antifungal therapy and the patients who received delayed antifungal therapy ( $25.5 \%$ and $23.1 \%)$. So we further analyzed demographic data, underlying diseases and clinical features between these two groups, the result showed that there were no significant differences between two groups in age $(p=0.33)$, $\operatorname{sex}(p=0.89)$, the number of underlying diseases $(p=0.32)$ and the number of severe clinical features $(p=0.96)$. The choice of antifungal agents between these two groups was further analyzed. In the appropriate antifungal therapy group, the rate of azoles use was $50.6 \%$, and the rate of echinocandin use was $30.3 \%$; while in the delayed antifungal therapy group, the rate of azoles use was $65.4 \%$, and the rate of echinocandin use was $17.3 \%$. The rate of echinocandin use was lower in the delayed antifungal therapy group than in the appropriate antifungal therapy group $(p=0.038)$. Is the lower rate of echinocandin use in the delayed antifungal therapy group one of the reasons that caused no significant difference in mortality between the two groups? Many studies have confirmed the important role of echinocandin in antifungal therapy. Echinocandin has been recommended as a first-line antifungal agent since 2009[10]. Many reports also have shown its non-inferiority or superiority to fluconazole [22, 23]. Therefore, we believe that the lower rate of echinocandin use in the delayed antifungal treatment group is unlikely to lead to a decrease in 30-day mortality rate. Based on the above analysis, we think that the timing of antifungal treatment has no significant impact on the 30-day mortality rate. Many clinicians currently administrate empirical antifungal agents; our study result may have an impact on their traditional view. Overuse use of antifungal drugs inevitably leads to waste of medical resources and rise of resistance. If taking empirical antifungal agents cannot improve mortality, we should take a second thought about it. There are several studies support our findings[7-9], they showed that severity of illness (APACHE-II Score) affected short-term survival 
in patients with candida infection, whereas the choice of initial antifungal agents did not affect short-term survival. However, some studies had different opinions on the timing of antifungal agents use. Bassetti's research showed that the use of antifungal agents within 48 hours of obtaining a positive candida blood culture result is an independent protective factor for mortality during hospitalization[6]; Similarly, Tedeschi's research showed that the administration of appropriate antifungal agents within 72 hours after blood culture was a protective factor for mortality during hospitalization[24]. However, due to the single-centered and the retrospective natures of their studies, those results need to be confirmed by more studies. Despite that, our results have shown similar the risk factors (for example, age, neutropenia, mechanical ventilation, etc.) and protective factor (antifungal therapy) for 30-day mortality to theirs.

Although our data have been collected from a large hospital in Shanghai, our study has some limitations, with the most significant one being its retrospective nature. The severity of illness score (such as APACHE II score), timing of CVC removal was not included because of missing data. Secondly, our study was monocentric and the results may not be applicable to other settings.

\section{Conclusion}

Our retrospective study showed that the incidence of Candida BSI has increased in the past 11 years in Shanghai. Although the percentage of non- $\mathrm{C}$. albicans has been increasing, the $\mathrm{C}$. albicans remained the most frequently isolated species. The mortality of patients with Candida bloodstream infection was quite high, especially for those in internal medicine wards. Antifungal therapy did improve short-term survival of patients with candida BSI. Whether we should initiate preemptive antifungal therapy or apply antifungal therapy after antifungal susceptibility test need further discussion.

\section{Declarations}

Ethics approval and consent to participate: The study was approved by the local institutional review board (Ruijin Hospital, Shanghai Jiaotong University, School of medicine) and written patient consent was not required because of the observational nature of this study.

Consent for publication: Not applicable.

Availability of data and materials: All data generated or analysed during this study are included in this published article and its supplementary information files.

Competing interests: The authors declare that they have no competing interests.

Funding: This work was supported by Program for Outstanding Medical Academic Leader, National Nature Science Foundation of China [81772107,81772040], Scientific and Technological Innovation Act Program of Science and Technology Commission of Shanghai Municipality [18411950900], Key Discipline Construction Project of Shanghai Municipal Commission of Health and Family Planning [2016ZB0206], Clinical Research Innovation Project of Shanghai Hospital Development Center [SHDC12017116] and Program of Shanghai Jiao Tong University School of Medicine [DLY201803] to Er-Zhen Chen. This work was also supported by Program of Shanghai Municipal Commission of Health and Family Planning [201640089] to Zhi-Tao Yang.

Authors' contributions: ZTY, EZC and EQM made substantial contributions to conception and design. ZTY, YJZ, LW, TX LZ and XYL participated in acquisition of data. ZTY, YJZ and YC drafted the manuscript. ZTY and EZC revised it critically.

Acknowledgements: We thank Ms. Wen-Jing ZHENG to produce the massive time first to the article English grammar, the usage and so on has carried on the revision.

\section{References}

1. Bitar D, Lortholary O, Le Strat Y, Nicolau J, Coignard B, Tattevin P, Che D, Dromer F: Population-based analysis of invasive fungal infections, France, 2001-2010. Emerging infectious diseases 2014, 20(7):1149-1155.

2. Jia X, Li C, Cao J, Wu X, Zhang L: Clinical characteristics and predictors of mortality in patients with candidemia: a six-year retrospective study. European Journal of Clinical Microbiology \& Infectious Diseases 2018, 37(9):1717-1724.

3. Marchetti O, Bille J, Fluckiger U, Eggimann P, Ruef C, Garbino J, Calandra T, Glauser M-P, Täuber MG, Pittet D et al: Epidemiology of Candidemia in Swiss Tertiary Care Hospitals: Secular Trends, 1991-2000. Clinical Infectious Diseases 2004, 38(3):311-320.

4. Wisplinghoff H, Bischoff T, Tallent SM, Seifert H, Wenzel RP, Edmond MB: Nosocomial Bloodstream Infections in US Hospitals: Analysis of 24,179 Cases from a Prospective Nationwide Surveillance Study. Clinical Infectious Diseases 2004, 39(3):309-317. 
5. Tiraboschi IN, Pozzi NC, Farias L, Garcia S, Fernandez NB: [Epidemiology, species, antifungal resistance and outcome of candidemia in a university hospital in Buenos Aires, Argentina for 16 years]. Revista chilena de infectologia : organo oficial de la Sociedad Chilena de Infectologia 2017, 34(5):431-440.

6. Bassetti M, Molinari MP, Mussap M, Viscoli C, Righi E: Candidaemia in internal medicine departments: the burden of a rising problem. Clinical Microbiology and Infection 2013, 19(6):E281-E284.

7. Parkins MD, Sabuda DM, Elsayed S, Laupland KB: Adequacy of empirical antifungal therapy and effect on outcome among patients with invasive Candida species infections. Journal of Antimicrobial Chemotherapy 2007, 60(3):613-618.

8. Taur Y, Cohen N, Dubnow S, Paskovaty A, Seo SK: Effect of antifungal therapy timing on mortality in cancer patients with candidemia. Antimicrobial agents and chemotherapy 2010, 54(1):184-190.

9. Grim SA, Berger K, Teng C, Gupta S, Layden JE, Janda WM, Clark NM: Timing of susceptibility-based antifungal drug administration in patients with Candida bloodstream infection: correlation with outcomes. Journal of Antimicrobial Chemotherapy 2011, 67(3):707-714.

10. Pappas PG, Kauffman CA, Andes D, Benjamin DK, Jr., Calandra TF, Edwards JE, Jr., Filler SG, Fisher JF, Kullberg B-J, Zeichner LO et al: Clinical Practice Guidelines for the Management Candidiasis: 2009 Update by the Infectious Diseases Society of America. Clinical Infectious Diseases 2009, 48(5):503-535.

11. Li F, Wu L, Cao B, Zhang Y, Li X, Liu Y: Surveillance of the prevalence, antibiotic susceptibility, and genotypic characterization of invasive candidiasis in a teaching hospital in China between 2006 to 2011. BMC infectious diseases 2013, 13:353.

12. Zhang L, Wang H, Xiao M, Kudinha T, Mao LL, Zhao HR, Kong F, Xu YC: The widely used ATB FUNGUS 3 automated readings in China and its misleading high MICs of Candida spp. to azoles: challenges for developing countries' clinical microbiology labs. PloS one 2014, 9(12):e114004.

13. Yang Z-T, Wu L, Liu X-Y, Zhou M, Li J, Wu J-Y, Cai Y, Mao E-Q, Chen E-Z, Lortholary O: Epidemiology, species distribution and outcome of nosocomial Candida spp. bloodstream infection in Shanghai. BMC infectious diseases 2014, 14:241-241.

14. Pappas PG, Kauffman CA, Andes DR, Clancy CJ, Marr KA, Ostrosky-Zeichner L, Reboli AC, Schuster MG, Vazquez JA, Walsh TJ et al: Clinical Practice Guideline for the Management of Candidiasis: 2016 Update by the Infectious Diseases Society of America. Clinical infectious diseases : an official publication of the Infectious Diseases Society of America 2016, 62(4):e1-50.

15. Ulu Kilic A, Alp E, Cevahir F, Ture Z, Yozgat N: Epidemiology and cost implications of candidemia, a 6-year analysis from a developing country. Mycoses 2017, 60(3):198-203.

16. Chapman B, Slavin M, Marriott D, Halliday C, Kidd S, Arthur I, Bak N, Heath CH, Kennedy K, Morrissey CO et al: Changing epidemiology of candidaemia in Australia. Journal of Antimicrobial Chemotherapy 2016, 72(4):1103-1108.

17. Sadeghi G, Ebrahimi-Rad M, Mousavi SF, Shams-Ghahfarokhi M, Razzaghi-Abyaneh M: Emergence of non-Candida albicans species: Epidemiology, phylogeny and fluconazole susceptibility profile. Journal de Mycologie Médicale 2018, 28(1):51-58.

18. Pfaller MA, Andes DR, Diekema DJ, Horn DL, Reboli AC, Rotstein C, Franks B, Azie NE: Epidemiology and outcomes of invasive candidiasis due to non-albicans species of Candida in 2,496 patients: data from the Prospective Antifungal Therapy (PATH) registry 2004-2008. PloS one 2014, 9(7):e101510.

19. Wu Z, Liu Y, Feng X, Liu Y, Wang S, Zhu X, Chen Q, Pan S: Candidemia: incidence rates, type of species, and risk factors at a tertiary care academic hospital in China. Int $J$ Infect Dis 2014, 22:4-8.

20. Maria S, Barnwal G, Kumar A, Mohan K, Vinod V, Varghese A, Biswas R: Species distribution and antifungal susceptibility among clinical isolates of Candida parapsilosis complex from India. Revista Iberoamericana de Micología 2018, 35(3):147-150.

21. Neji S, Hadrich I, Trabelsi H, Abbes S, Cheikhrouhou F, Sellami H, Makni F, Ayadi A: Virulence factors, antifungal susceptibility and molecular mechanisms of azole resistance among Candida parapsilosis complex isolates recovered from clinical specimens. Journal of biomedical science 2017, 24(1):67.

22. Lin K-Y, Chen P-Y, Chuang Y-C, Wang J-T, Sun H-Y, Sheng W-H, Chen Y-C, Chang S-C: Effectiveness of echinocandins versus fluconazole for treatment of persistent candidemia: A time-dependent analysis. Journal of Infection 2018, 77(3):242-248.

23. Reboli AC, Rotstein C, Pappas PG, Chapman SW, Kett DH, Kumar D, Betts R, Wible M, Goldstein BP, Schranz J et al: Anidulafungin versus Fluconazole for Invasive Candidiasis. New England Journal of Medicine 2007, 356(24):2472-2482.

24. Tedeschi S, Tumietto F, Giannella M, Bartoletti M, Cristini F, Cioni G, Ambretti S, Carretto E, Sambri V, Sarti M et al: Epidemiology and outcome of candidemia in internal medicine wards: A regional study in Italy. European Journal of Internal Medicine 2016, 34:39-44.

\section{Tables}

Table 1. Demographic of patients with candida bloodstream infection and distribution of Candida species 


\begin{tabular}{|c|c|c|c|c|c|c|c|c|c|}
\hline & $\begin{array}{l}\text { C. } \\
\text { albicans }\end{array}$ & $\begin{array}{l}\text { C. } \\
\text { Parapsilosis }\end{array}$ & $\begin{array}{l}\text { C. } \\
\text { Tropicalis }\end{array}$ & $\begin{array}{l}\text { C. } \\
\text { glabrata }\end{array}$ & $\begin{array}{l}\text { C. } \\
\text { guilliermondii }\end{array}$ & $\begin{array}{l}\text { C. } \\
\text { sake }\end{array}$ & $\begin{array}{l}\text { C. } \\
\text { krusei }\end{array}$ & $\begin{array}{l}\text { Other } \\
\text { Candida spp. }\end{array}$ & Total \\
\hline & $(n=141)$ & $(\mathrm{n}=87)$ & $(\mathrm{n}=69)$ & $(\mathrm{n}=48)$ & $(\mathrm{n}=20)$ & $(n=8)$ & $(n=5)$ & $(\mathrm{n}=15)$ & $(\mathrm{n}=393)$ \\
\hline Age (year) & $65.2 \pm 14.5$ & $53.2 \pm 20.3$ & $50.5 \pm 19.5$ & $60.7 \pm 17.5$ & $50.5 \pm 19.4$ & $52 \pm 20.6$ & $40.8 \pm 27.1$ & $52.6 \pm 20.0$ & $57.6 \pm 19.0$ \\
\hline Male[n,\%[ & $102(73.4)$ & $60(69.0)$ & $51(73.9)$ & $44(91.7)$ & $14(70)$ & $8(100)$ & $2(40)$ & $11(73.3)$ & $292(74.3)$ \\
\hline \multicolumn{10}{|l|}{ Origin $\llbracket n, \% \square$} \\
\hline Internal medicine ward & $15(10.6)$ & $19(21.8)$ & $27(39.1)$ & $7(14.6)$ & $3(15)$ & $0(0)$ & $3(60)$ & $4(26.7)$ & 78(19.8) \\
\hline Surgical ward & $61(43.3)$ & $32(36.8)$ & $16(23.2)$ & $15(31.3)$ & $10(50)$ & $6(75)$ & $0(0)$ & $8(53.3)$ & $148(37.7)$ \\
\hline ICU & $65(46.1)$ & $36(41.4)$ & $26(37.7)$ & $26(54.1)$ & $7(35)$ & $2(25)$ & $2(40)$ & $3(20)$ & $167(42.5)$ \\
\hline $\begin{array}{l}\text { Time from admission to } \\
\text { infection (d) }\end{array}$ & $30.6 \pm 35.3$ & $48.4 \pm 56.2$ & $37.7 \pm 32.4$ & $27.5 \pm 19.1$ & $35.9 \pm 52.5$ & $120.6 \pm 242.7$ & $64.2 \pm 68.6$ & $21.5 \pm 13.6$ & $37.6 \pm 53.1$ \\
\hline Length of hospital stay (d) & $56.0 \pm 54.9$ & $83.2 \pm 78.1$ & $71.5 \pm 56.1$ & $67.1 \pm 72.7$ & $97.7 \pm 142.6$ & $178.5 \pm 281.7$ & $92.4 \pm 88.5$ & $52.7 \pm 101.5$ & $71.1 \pm 82.8$ \\
\hline Turnaround Time (d) & $4.3 \pm 1.9$ & $4.5 \pm 1.1$ & $3.8 \pm 1.1$ & $4.8 \pm 1.3$ & $4.3 \pm 1.2$ & $4.5 \pm 1.6$ & $4.4 \pm 1.5$ & $5.9 \pm 1.8$ & $4.4 \pm 1.5$ \\
\hline
\end{tabular}

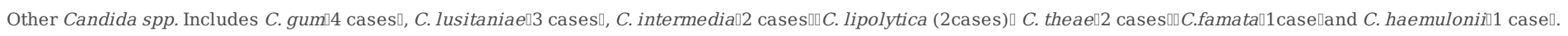

Abbreviation: ICU, intensive care unit

Table2. Underlying disease and clinical feature of Candida bloodstream infection $(n, \%)$

\begin{tabular}{|c|c|c|c|c|c|c|c|c|c|}
\hline & c. & C. Parapsilosis & C. Tropicalis & C. glabrata & C. guilliermondii & C. & C. & OtherCandida spp. & Total \\
\hline & albicans & & & & & sake & krusei & & \\
\hline & $(\mathrm{n}=141)$ & $(\mathrm{n}=87)$ & $(\mathrm{n}=69)$ & $(n=48)$ & $(\mathrm{n}=20)$ & $(\mathrm{n}=8)$ & $(\mathrm{n}=5)$ & $(\mathrm{n}=15)$ & $(\mathrm{n}=393)$ \\
\hline \multicolumn{10}{|l|}{ Underlying disease } \\
\hline Solid tumor & $47(33.3)$ & $26(29.9)$ & 13(18.8) & $17(35.4)$ & $6(30)$ & $2(25)$ & $0(0)$ & $7(46.7)$ & $118(30)$ \\
\hline Hematologic malignancy & $8(5.7)$ & $6(6.9)$ & $24(34.8)$ & $2(4.2)$ & $2(10)$ & $1(12.5)$ & $3(60)$ & $2(13.3)$ & $48(12.2)$ \\
\hline Diabetes mellitus & $33(23.4)$ & $21(24.1)$ & $8(11.6)$ & $9(18.8)$ & $1(5)$ & $1(12.5)$ & $0(0)$ & $4(26.7)$ & $77(19.6)$ \\
\hline Chronic cardiac disease & $55(39)$ & $22(25.3)$ & $16(23.2)$ & $18(37.5)$ & $6(30)$ & $3(37.5)$ & $2(40)$ & $2(13.3)$ & $124(31.6)$ \\
\hline Chronic pulmonary disease & $26(18.4)$ & $9(10.3)$ & $5(7.2)$ & $6(12.5)$ & $1(5)$ & $1(12.5)$ & $1(20)$ & $3(20)$ & $52(13.2)$ \\
\hline Chronic renal failure & 18(12.8) & $6(6.9)$ & $6(8.7)$ & $5(10.4)$ & $5(25)$ & $0(0)$ & $0(0)$ & $2(13.3)$ & $42(10.7)$ \\
\hline Skin barrier compromised & $5(3.5)$ & $9(10.3)$ & $5(7.2)$ & $1(2.1)$ & $3(15)$ & $3(37.5)$ & $0(0)$ & $0(0)$ & $26(6.6)$ \\
\hline Prior surgical intervention ( $<1$ month) & $97(68.8)$ & $48(55.2)$ & $36(52.2)$ & $30(62.5)$ & $15(75)$ & $6(75)$ & $2(40)$ & 10(66.7) & $244(62.1)$ \\
\hline Corticosteroid use & $11(7.8)$ & 13(14.9) & $12(17.4)$ & $9(18.8)$ & $4(20)$ & $0(0)$ & $3(60)$ & $2(13.3)$ & $54(13.7)$ \\
\hline Prior use of antifungal agents ( $<6$ months) & $20(14.2)$ & 19(21.8) & $24(34.8)$ & $11(22.9)$ & $8(40)$ & $1(12.5)$ & $4(80)$ & $1(6.7)$ & $88(22.4)$ \\
\hline \multicolumn{10}{|l|}{ Severity of clinical feature } \\
\hline Fever $\left(\mathrm{T}>38.2^{\circ} \mathrm{C}\right)$ & $114(80.9)$ & $63(72.4)$ & $60(87)$ & $34(70.8)$ & $16(80)$ & $6(75)$ & $3(60)$ & 13(86.7) & 309(78.6) \\
\hline Parenteral nutrition & $71(50.4)$ & $42(48.3)$ & $28(40.6)$ & $23(47.9)$ & $5(25)$ & $3(37.5)$ & $2(40)$ & $6(40)$ & $180(45.8)$ \\
\hline Mechanical ventilation & $58(41.1)$ & $31(35.6)$ & $23(33.3)$ & $25(52.1)$ & $5(25)$ & $2(25)$ & $2(40)$ & $1(6.7)$ & $147(37.4)$ \\
\hline Renal replacement therapy & $17(12.1)$ & $10(11.5)$ & $9(13)$ & $7(14.6)$ & $5(25)$ & $0(0)$ & $1(20)$ & $0(0)$ & $49(12.5)$ \\
\hline Central venous catheter & $121(85.8)$ & $67(77)$ & $49(71)$ & 43(89.6) & $17(85)$ & $5(62.5)$ & $4(80)$ & $10(66.7)$ & $316(80.4)$ \\
\hline Neutropenia & $4(2.8)$ & $8(9.2)$ & $22(31.9)$ & $1(2.1)$ & $2(10)$ & $0(0)$ & $3(60)$ & $2(13.3)$ & $42(10.7)$ \\
\hline 28-day mortality & $54(38.3)$ & $16(18.4)$ & $19(27.5)$ & $13(27.1)$ & $3(15)$ & $1(12.5)$ & $1(20)$ & $5(33.3)$ & $112(28.5)$ \\
\hline
\end{tabular}




\begin{tabular}{|c|c|c|c|c|c|}
\hline \multirow[t]{2}{*}{ 28-day outcome } & \multirow[b]{2}{*}{ Survival ( $n=281$ ) } & \multirow[b]{2}{*}{ Death $(n=112)$} & \multirow[b]{2}{*}{$P$ value } & \multicolumn{2}{|l|}{ Multivariate analysis } \\
\hline & & & & HR $(95 \% \mathrm{CI})$ & $P$ value \\
\hline Gender, male [n (\%)] & $216(76.9)$ & $76(67.9)$ & 0.065 & - & - \\
\hline Underlying disease & & & & - & - \\
\hline Solid tumor [n (\%)] & $91(32.4)$ & $27(24.1)$ & 0.106 & - & - \\
\hline Chronic Cardiac disease [n (\%)] & $72(25.6)$ & $52(46.4)$ & $<0.01$ & - & 0.105 \\
\hline Chronic Pulmonary disease [n (\%)] & $34(12.1)$ & 18(16.1) & 0.294 & - & - \\
\hline Chronic renal failure [n (\%)] & $20(7.1)$ & $22(19.6)$ & $<0.01$ & $2.018(1.234-3.299)$ & 0.005 \\
\hline Skin barrier compromised [n (\%)] & $24(8.5)$ & $2(1.8)$ & 0.015 & - & 0.308 \\
\hline Prior surgical intervention (<1month) [n (\%)] & 182(64.8) & $62(55.4)$ & 0.083 & - & - \\
\hline Corticosteroid use [n (\%)] & $40(14.2)$ & $14(12.5)$ & 0.652 & - & - \\
\hline Parenteral nutrition [n (\%)] & 124(44.1) & $56(50)$ & 0.292 & - & - \\
\hline Mechanical ventilation $[\mathrm{n}(\%)]$ & $89(31.7)$ & $58(51.8)$ & $<0.01$ & $1.950(1.307-2.912)$ & 0.001 \\
\hline Renal replacement therapy [n (\%)] & $32(11.4)$ & $17(15.2)$ & 0.305 & - & - \\
\hline Central venous catheter [n (\%)] & $227(80.8)$ & $89(79.5)$ & 0.766 & - & - \\
\hline Neutropenia [n (\%)] & $24(8.5)$ & 18(16.1) & 0.029 & $4.347(2.462-7.675)$ & $<0.001$ \\
\hline Antifungal therapy & $224(74.9)$ & $75(25.1)$ & 0.007 & $0.502(0.294-0.857)$ & 0.006 \\
\hline No treatment & $57(60.6)$ & $37(39.4)$ & & & \\
\hline
\end{tabular}




\begin{tabular}{|c|c|c|c|c|c|c|c|c|c|}
\hline & C.albicans $(\mathrm{n}=134)$ & C.parapsilosis(n=86) & C.tropicalis $(\mathrm{n}=67)$ & C.glabrata $(\mathrm{n}=47)$ & C.krusei $(\mathrm{n}=5)$ & C.sake $(\mathrm{n}=8)$ & $\begin{array}{l}\text { C.guilliermondii } \\
(\mathrm{n}=19)\end{array}$ & $\begin{array}{l}\text { Other } \\
\text { Candida } \\
\text { spp. }(\mathrm{n}=12)\end{array}$ & $\begin{array}{l}\text { Total } \\
\square \mathrm{n}=378 \square\end{array}$ \\
\hline \multicolumn{10}{|c|}{ Fluconazole } \\
\hline S & $126(94)$ & 77(89.5) & $35(52.2)$ & $0(0)$ & $0(0)$ & $8(100)$ & $13(68.4)$ & $9(75.0)$ & $\begin{array}{l}268 \\
\square 70.9 \square\end{array}$ \\
\hline SDD & $1(0.8)$ & $6(7.0)$ & $3(4.5)$ & $44(93.6)$ & $0(0)$ & $0(0)$ & $0(0)$ & $0(0)$ & $\begin{array}{l}54 \\
\square 14.3 \square\end{array}$ \\
\hline $\mathbf{R}$ & $7(5.2)$ & $3(3.5)$ & $29(43.3)$ & $3(6.4)$ & $5(100)$ & $0(0)$ & $6(31.6)$ & $3(25.0)$ & $\begin{array}{l}56 \\
\square 14.8 \square\end{array}$ \\
\hline \multicolumn{10}{|c|}{ Itraconazole } \\
\hline $\mathrm{s}$ & $110(82.1)$ & $75(87.2)$ & 25 (37.3) & $0(0)$ & $0(0)$ & $8(100)$ & $6(31.6)$ & $9(75.0)$ & $\begin{array}{l}233 \\
\square 61.6 \square\end{array}$ \\
\hline SDD & $6(4.5)$ & $7(8.1 \square$ & $4(6.0)$ & $40(85.1)$ & $2(40.0)$ & $0(0)$ & 7 (36.8) & $0(0)$ & $\begin{array}{l}66 \\
\square 17.5 \square\end{array}$ \\
\hline $\mathbf{R}$ & $18(13.4)$ & $4 \square 4.7 \square$ & $38(56.7)$ & $7(14.9)$ & $3(60.0)$ & $0(0)$ & $6(31.6)$ & $3(25.0)$ & $\begin{array}{l}79 \\
\square 20.9 \square\end{array}$ \\
\hline \multicolumn{10}{|c|}{ Voriconazole } \\
\hline $\mathrm{s}$ & 125 (93.3) & 79 (91.9) & $41(61.2)$ & 45 (95.8) & $4(80.0)$ & $8(100)$ & $12(63.2)$ & 11 (91.7) & $\begin{array}{l}325 \\
\square 86.0 \square\end{array}$ \\
\hline SDD & $0(0)$ & $2(2.3)$ & $2(3.0)$ & $1(2.1)$ & $1(2.0)$ & $0(0)$ & $3(15.8)$ & $0(0)$ & $9 \square 2.4 \square$ \\
\hline $\mathbf{R}$ & $9(6.7)$ & $5(5.8)$ & $24(35.8)$ & $1(2.1)$ & $0(0)$ & $0(0)$ & $4(21.0)$ & $1(8.3)$ & $\begin{array}{l}44 \\
\square 11.6 \square\end{array}$ \\
\hline \multicolumn{10}{|c|}{$\begin{array}{l}\text { Amphotericin } \\
\text { B }\end{array}$} \\
\hline $\mathrm{s}$ & 133 (99.3) & 83 (96.5) & $67(100)$ & 47 (100) & $5(100)$ & $8(100)$ & $18(94.7)$ & 11 (91.7) & $\begin{array}{l}372 \\
\square 98.4 \square\end{array}$ \\
\hline $\mathbf{R}$ & $1(0.7)$ & $3(3.5)$ & $0(0)$ & $0(0)$ & $0(0)$ & $0(0)$ & $1(5.3)$ & $1(8.3)$ & $6 \square 1.6 \square$ \\
\hline \multicolumn{10}{|c|}{ Flucytosin } \\
\hline $\mathrm{s}$ & 132 (98.5) & 85 (98.8) & $65(97.0)$ & $46(97.9)$ & $1(20.0)$ & $8(100)$ & $9(47.4)$ & $12(100)$ & $\begin{array}{l}358 \\
\square 94.7 \square\end{array}$ \\
\hline $\mathbf{R}$ & $2(1.5)$ & $1(1.2)$ & $2(3.0)$ & $1(2.1)$ & $4(80.0)$ & $0(0)$ & $10(52.6]$ & $0(0)$ & $20 \square 5.3 \rrbracket$ \\
\hline
\end{tabular}

15 Candida spp. isolats did not have susceptibility test, C. albicans (7), C. parapsilosis (2), C. tropicalis (2), and glabrata, theae, gum,haemulonii each.

\section{Figures}


Fig1. Distribution of Candida spp. during study period(From 2008 to 2018)

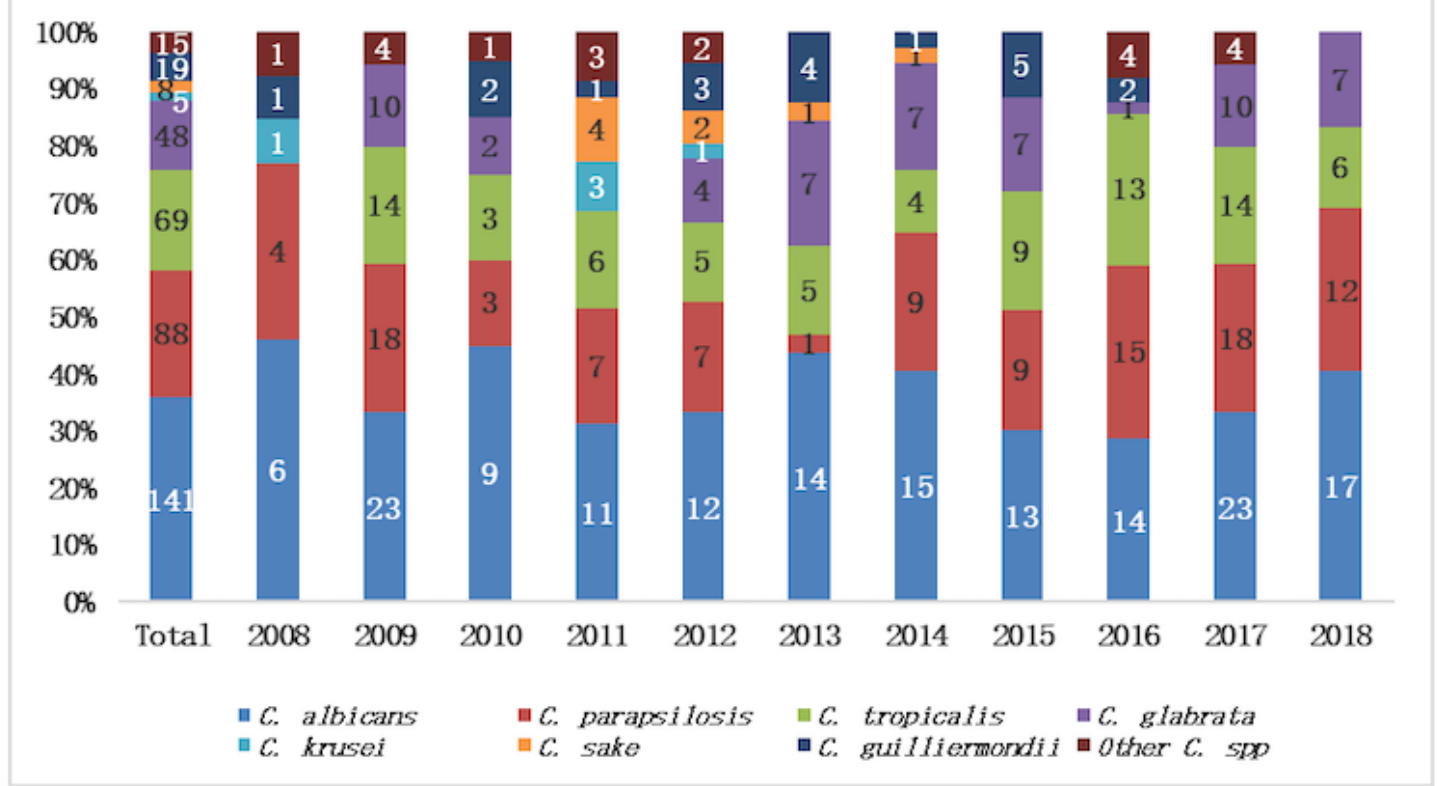

Figure 1 
Fig2. Distribution of Candida spp.

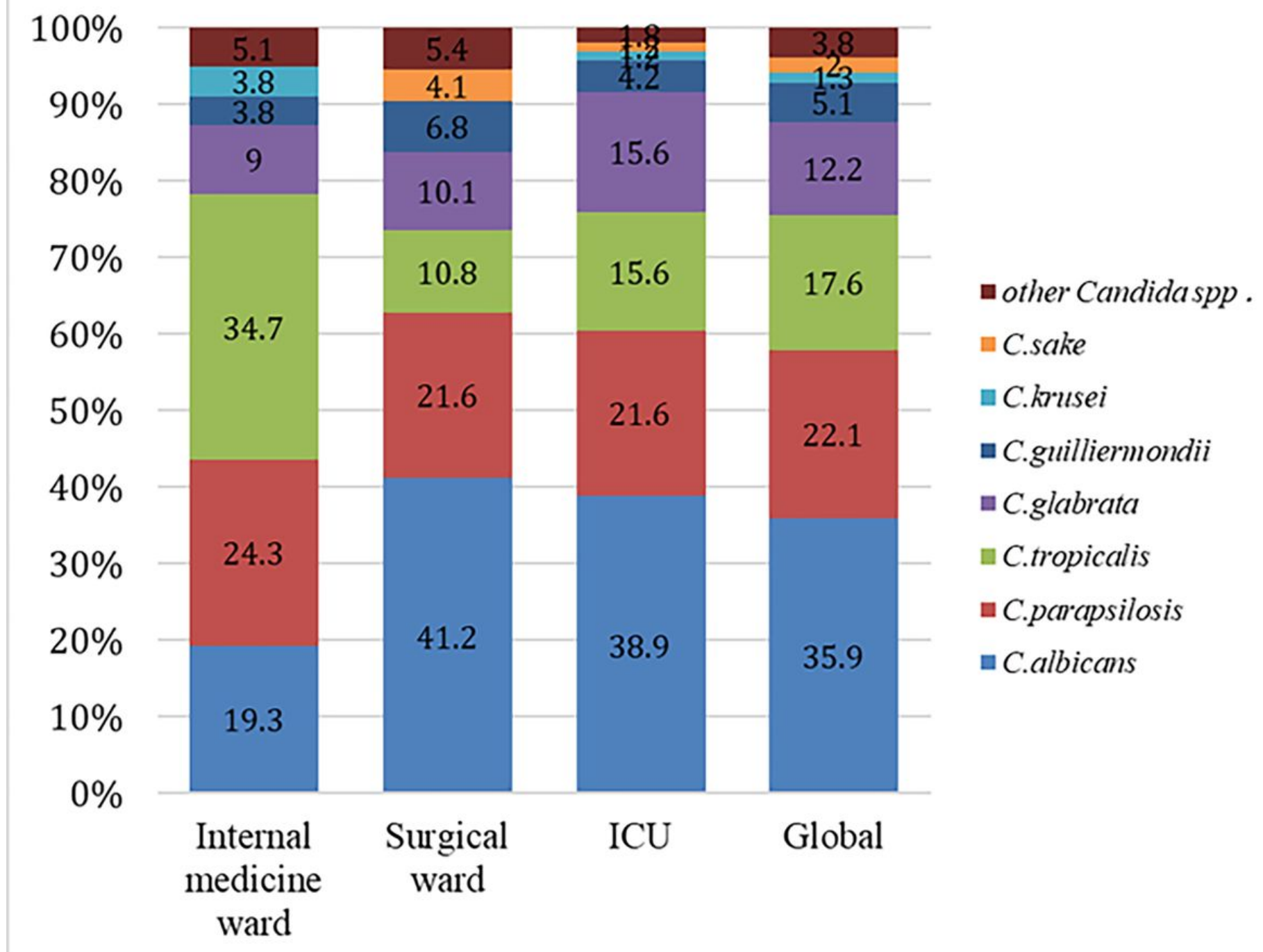

Figure 2 


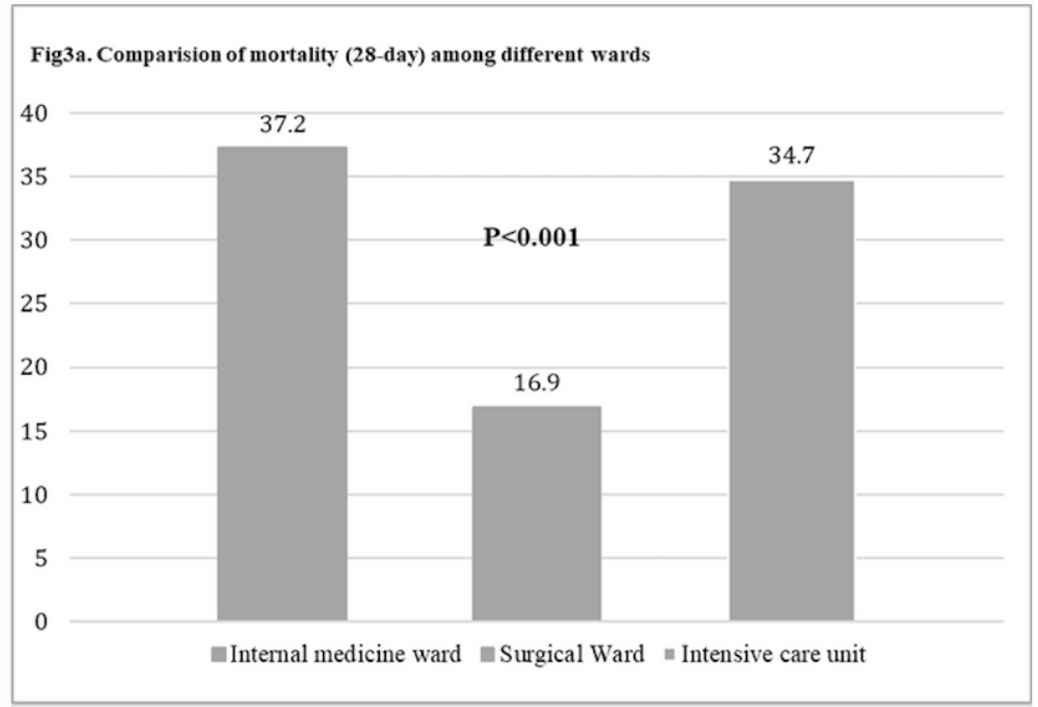

Fig3b. Relationship between hospital mortality (28-day) and the timing of antifungal treatment.

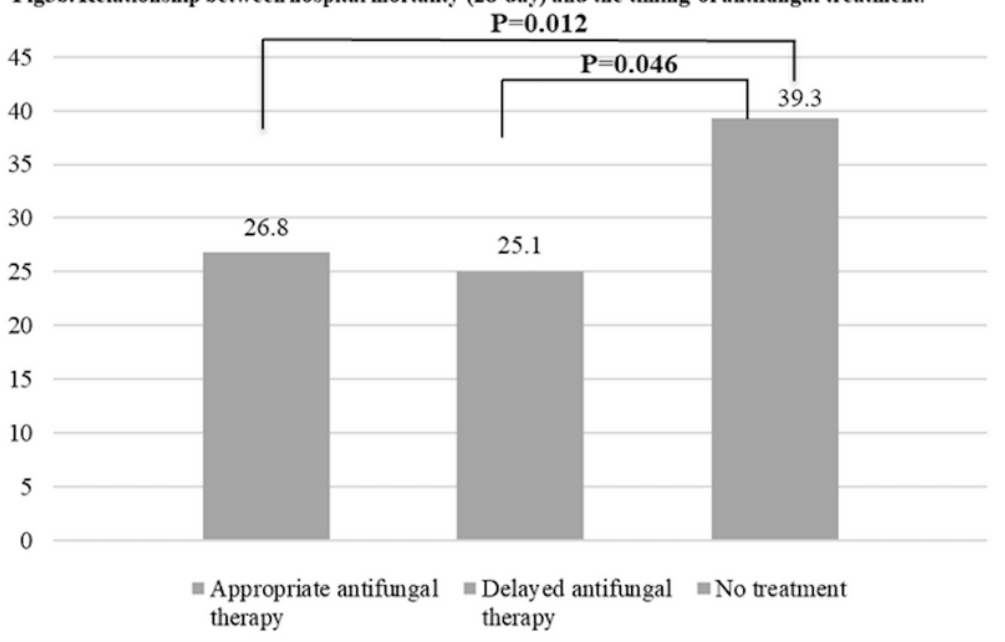

Figure 3 